\title{
THE
}

\section{Routh-Hurwitz Criterion in the Examination of Eigenvalues of a System of Nonlinear Ordinary Differential Equations}

\author{
Edmund X. DeJesus \\ Charles Kaufman \\ University of Rhode Island, ckaufman@uri.edu
}

Follow this and additional works at: https://digitalcommons.uri.edu/phys_facpubs

Terms of Use

All rights reserved under copyright.

\section{Citation/Publisher Attribution}

DeJesus, E. X., \& Kaufman, C. (1987). Routh-Hurwitz criterion in the examination of eigenvalues of a system of nonlinear ordinary differential equations. Physical Review A, 35(12), 528-5290. doi: 10.1103/ PhysRevA.35.5288

Available at: http://dx.doi.org/10.1103/PhysRevA.35.5288

This Article is brought to you for free and open access by the Physics at DigitalCommons@URI. It has been accepted for inclusion in Physics Faculty Publications by an authorized administrator of DigitalCommons@URI. For more information, please contact digitalcommons-group@uri.edu. 


\title{
Routh-Hurwitz criterion in the examination of eigenvalues of a system of nonlinear ordinary differential equations
}

\author{
Edmund X. DeJesus \\ Division of Science, College of Basic Studies, Boston University, Boston, Massachusetts 02215 \\ Charles Kaufman \\ Department of Physics, University of Rhode Island, Kingston, Rhode Island 02881
}

(Received 27 January 1987)

\begin{abstract}
In stability analysis of nonlinear systems, the character of the eigenvalues of the Jacobian matrix (i.e., whether the real part is positive, negative, or zero) is needed, while the actual value of the eigenvalue is not required. We present a simple algebraic procedure, based on the Routh-Hurwitz criterion, for determining the character of the eigenvalues without the need for evaluating the eigenvalues explicitly. This procedure is illustrated for a system of nonlinear ordinary differential equations we have studied previously. This procedure is simple enough to be used in computer code, and, more importantly, makes the analysis possible even for those cases where the secular equation cannot be solved.
\end{abstract}

\section{INTRODUCTION}

When studying systems of nonlinear ordinary differential equations, it is often useful to examine the eigenvalues of the associated linearized system. For example, consider the system of nonlinear ordinary differential equations given by

$$
\dot{W}=H \cdot W+G(W),
$$

where $W=W(t)$ is a column $n$ vector, $H$ is an $n \times n$ matrix, and $G(W)$ is a nonlinear vector-valued function. $G(W)$ is defined such that $G(W) /\|W\|$ is continuous and vanishes for $W \rightarrow$ zero. The stability of solutions to a system (1) satisfying these conditions can be determined using the eigenvalues of $H:^{1}$

(1) The equilibrium solution $W(t) \equiv 0$ is asymptotically stable if all the eigenvalues of $H$ have negative real part.

(2) The equilibrium solution $W(t) \equiv 0$ is unstable if at least one eigenvalue of $H$ has positive real part.

(3) The stability of the equilibrium solution $W(t) \equiv 0$ cannot be determined from $H$ alone if all the eigenvalues of $H$ have a real part less than or equal to 0 but at least one eigenvalue of $H$ has a zero real part.

Thus, we do not really need to know the numerical values of the eigenvalues: All we need to know is the character of each eigenvalue. (By character we mean whether the real part of the eigenvalue is positive, negative, or zero.) We note also that in practice it may be difficult, or impossible, to calculate the actual values of the eigenvalues. Finding the values of the eigenvalues is equivalent to finding the zeros of an $n$ th-degree polynomial. For $n>5$ there exists no general procedure for determining the zeros of an $n$ th-degree polynomial (and, equivalently, the values of the eigenvalues), except for special cases.

We outline here a simple algebraic procedure for quick- ly determining the character of the eigenvalues. The procedure is based on the Routh-Hurwitz criterion for determining the character of zeros of an $n$ th-degree polynomial. $^{2}$

We illustrate the procedure by applying it to a system of nonlinear ordinary differential equations that we have used previously ${ }^{3}$ to model fluid flow within a boundary layer along a flat plate. Briefly, a truncation of the Fourier series solution to the Navier-Stokes equations yields a system of nonlinear ordinary differential equations:

$$
\begin{aligned}
& \dot{A}=d_{1} A+d_{2} B \cdot C, \\
& \dot{B}=e_{1} B+e_{2} A \cdot C+e_{3} C, \\
& \dot{C}=f_{1} C+f_{2} A \cdot B,
\end{aligned}
$$

where $A, B$, and $C$ are functions of time $t$. The functions $A, B$, and $C$ are proportional to the amplitudes of the terms of the truncated Fourier series. In (2),

$$
\begin{aligned}
& d_{1}=-l^{2} / R, \quad d_{2}=\frac{2 l^{3}}{k\left(k^{2}+l^{2}\right)}, \\
& e_{1}=-k^{2} / R, \quad e_{2}=\frac{-2 k^{3}}{1\left(k^{2}+l^{2}\right)}, \quad e_{3}=\frac{-2 k m}{k^{2}+l^{2}}, \\
& f_{1}=-\left(k^{2}+l^{2}\right) / R, \quad f_{2}=\frac{k^{2}-l^{2}}{2 k l},
\end{aligned}
$$

where $k$ and $l$ are constants related to the wave numbers of terms of the Fourier series, $m$ is a constant related to the assumed boundary-layer flow along the plate, and $R$ is a variable. $R$ is the Reynolds number of the fluid. In our previous work we used $k=0.62, \quad l=0.07$, and $m=-0.064$, and examined the behavior of system (2) as we varied $R$ in the range 1 to 40 . 


\section{PROCEDURE}

Step $A$. We change the system (2) so that it resembles system (1). Specifically, we want $W \equiv 0$ to be the equilibrium solution; also we want to split the system into linear and nonlinear parts corresponding to the matrix $H$ and the function $G$.

(1) Identify the equilibrium points of (2), that is, those points where $\dot{A}, \dot{B}$, and $\dot{C}$ are zero. In system (2) there are five equilibrium points, which we represent as $P_{i}=\left(A_{i}, B_{i}, C_{i}\right)$ for $i=0,1,2,3,4$. These five equilibrium points are

$P_{0}: A_{0}=0, B_{0}=0, C_{0}=0$,

$P_{1}$ :

$$
\begin{aligned}
& A_{1}=\frac{-m l}{2 k^{2}}+\frac{1}{2}\left[\frac{m^{2} l^{2}}{k^{4}}+\frac{4 l^{2}\left(k^{2}+l^{2}\right)^{2}}{R^{2}\left(l^{2}-k^{2}\right)}\right]^{1 / 2}, \\
& B_{1}=\frac{k\left(k^{2}+l^{2}\right)}{R}\left[\frac{1}{\left(k^{2}-l^{2}\right)}\right)^{1 / 2}, \\
& C_{1}=\frac{A_{1}}{2 l}\left(k^{2}-l^{2}\right)^{1 / 2},
\end{aligned}
$$

$P_{2}: \quad A_{2}=A_{1}, \quad B_{2}=-B_{1}, \quad C_{2}=-C_{1}$,

$P_{3}$ :

$$
\begin{gathered}
A_{3}=\frac{-m l}{2 k^{2}}-\frac{1}{2}\left(\frac{m^{2} l^{2}}{k^{4}}+\frac{4 l^{2}\left(k^{2}+l^{2}\right)^{2}}{R^{2}\left(l^{2}-k^{2}\right)}\right)^{1 / 2}, \\
B_{3}=B_{1}, \quad C_{3}=\frac{A_{3}}{2 l}\left(k^{2}-l^{2}\right)^{1 / 2} \\
P_{4}: A_{4}=A_{3}, \quad B_{4}=-B_{1}, \quad C_{4}=-C_{3} .
\end{gathered}
$$

Notice that for $R$ less than a critical value $R_{c}$, only $P_{0}$ is real and $P_{1-4}$ are all complex. For $R$ equal to $R_{c}$ all the equilibrium points are real, but $P_{1} \equiv P_{3}$ and $P_{2} \equiv P_{4}$. For $R$ greater than $R_{c}$ all the equilibrium points are real and distinct. The value of $R_{c}$ can easily be computed by setting the radicand in $A_{1}$ or $A_{3}$ equal to zero; thus

$$
R_{c}=\left|\frac{2\left(k^{2}+l^{2}\right) k^{2}}{m\left(k^{2}-l^{2}\right)^{1 / 2}}\right|=7.59 \cdots
$$

for our values of $k, l$, and $m$.

(2) Transform system (2) so that $W \equiv 0$ is the equilibrium solution. This can be done quite simply as follows:

$$
\left.\begin{array}{r}
X=A-A_{i}, \\
Y=B-B_{i}, \\
Z=C-C_{i}
\end{array}\right\} \text { for } i=0,1,2,3,4 .
$$

(3) Recast system (2) in terms of the transformation (4). This results in a new nonlinear system:

$$
\begin{aligned}
& \dot{X}=d_{1} X+d_{2} C_{i} Y+d_{2} B_{i} Z+d_{2} Y \cdot Z, \\
& \dot{Y}=e_{2} C_{i} X+e_{1} Y+\left(e_{2} A_{i}+e_{3}\right) Z+e_{2} X \cdot Z, \\
& \dot{Z}=f_{2} B_{i} X+f_{2} A_{i} Y+f_{1} Z+f_{2} X \cdot Y .
\end{aligned}
$$

Comparing system (5) with system (1), we can identify $W=(X, Y, Z), G(W)=\left(d_{2} Y \cdot Z, e_{2} X \cdot Z, f_{2} X \cdot Y\right)$ and

$$
H=\left(\begin{array}{ccc}
d_{1} & d_{2} C_{i} & d_{2} B_{i} \\
e_{2} C_{i} & e_{1} & e_{2} A_{i}+e_{3} \\
f_{2} B_{i} & f_{2} A_{i} & f_{1}
\end{array}\right)
$$

as desired. Since system (5) is in the form of system (1), we could now determine the stability of the equilibrium solutions by examining the eigenvalues of the matrix $H$.

Step B. Start determining the eigenvalues of the matrix $H$. We do this in the usual way, by setting $\operatorname{det}(H-\underline{\lambda} I)=0$. This yields the characteristic equation

$$
\begin{aligned}
0= & \underline{\lambda}^{3}+\left(-d_{1}-e_{1}-f_{1}\right) \underline{\lambda}^{2} \\
& +\left(d_{1} e_{1}+d_{1} f_{1}+e_{1} f_{1}-e_{2} f_{2} A_{i}^{2}-d_{2} f_{2} B_{i}^{2}-d_{2} e_{2} C_{i}^{2}-e_{3} f_{2} A_{i}\right) \underline{\underline{\lambda}} \\
& +\left(d_{1} e_{3} f_{2} A_{i}-d_{1} e_{1} f_{1}+d_{1} e_{2} f_{2} A_{i}^{2}+d_{2} e_{1} f_{2} B_{i}^{2}+d_{2} e_{2} f_{1} C_{i}^{2}\right. \\
& \left.-d_{2} e_{2} f_{2} A_{i} B_{i} C_{i}-d_{2} e_{2}^{2} A_{i} B_{i} C_{i}-d_{2} e_{3} f_{2} B_{i} C_{i}\right) .
\end{aligned}
$$

Using the definitions of $d_{1}, d_{2}, e_{1}, e_{2}, e_{3}, f_{1}, f_{2}$, and the possible choices for $A_{i}, B_{i}$, and $C_{i}$ in Eq. (7) yields

$$
\begin{aligned}
0= & \underline{\lambda}^{3}+\left(\frac{2\left(k^{2}+l^{2}\right)}{R}\right) \underline{\lambda}^{2}+\left(\frac{m l\left(l^{2}-k^{2}\right) A_{i}}{\left(k^{2}+l^{2}\right)^{2}}\right) \underline{\lambda} \\
& +\frac{\left(k^{2}-l^{2}\right) A_{i}}{R\left(k^{2}+l^{2}\right)}\left(4 k^{2} A_{i}+2 m l\right) .
\end{aligned}
$$

This is the characteristic equation one would ordinarily solve to determine the eigenvalues of $H$. Equation (8) is a cubic equation in $\underline{\lambda}$. A general solution to the cubic equation exists, ${ }^{4}$ but is very complicated. To obtain a general solution to (8), we would take the coefficients of the $\underline{\lambda}$ terms and substitute them into the general solution to the cubic. The result is an extremely complicated equation which obscures, rather than elucidates, the character of the eigenvalues as the value of $R$ changes. Recall that we only desire to know the character of the eigenvalues. We do not need to solve (8) for $\underline{\lambda}$.

Step C. Use the Routh-Hurwitz criterion to determine the character of the solutions to (8). We first write our $n$ th-degree polynomial in the form

$$
0=a_{0} \underline{\lambda}^{n}+a_{1} \underline{\lambda}^{n-1}+\cdots+a_{j} \underline{\lambda}^{n-j}+\cdots+a_{n} .
$$

Comparing this form to equation (8), we can identify 


$$
\begin{aligned}
& a_{0}=1, \\
& a_{1}=\frac{2\left(k^{2}+l^{2}\right)}{R}, \\
& a_{2}=\frac{m l\left(l^{2}-k^{2}\right) A_{i}}{\left(k^{2}+l^{2}\right)^{2}}, \\
& a_{3}=\frac{\left(k^{2}-l^{2}\right) A_{i}}{R\left(k^{2}+l^{2}\right)}\left(4 k^{2} A_{i}+2 m l\right) .
\end{aligned}
$$

Next we form the numbers $T_{0}, T_{1}, T_{2}, T_{3}$ as

$$
\begin{aligned}
T_{0} & =a_{0}=1, \\
T_{1} & =a_{1}=\frac{2\left(k^{2}+l^{2}\right)}{R}, \\
T_{2} & =\left|\begin{array}{ll}
a_{1} & a_{0} \\
a_{3} & a_{2}
\end{array}\right|=a_{1} a_{2}-a_{3} a_{0}=\frac{4 k^{2} l^{2}\left(k^{2}+l^{2}\right)}{R^{3}}, \\
T_{3} & =\left|\begin{array}{ccc}
a_{1} & a_{0} & 0 \\
a_{3} & a_{2} & a_{1} \\
0 & 0 & a_{3}
\end{array}\right|=a_{3} T_{2} .
\end{aligned}
$$

(More generally, $T_{k}$ is the determinant of the $k \times k$ matrix, $M_{i j}$, for $k \leq n$. The matrix $M_{i j}$, is formed using this rule: if $2 i-j<0$ or $2 i-j>n$, then $M_{i j}=0$; otherwise, $M_{i j}=a_{2 i-j}$.) Next we construct the sequence $T_{0}, T_{1}, T_{2} / T_{1}, T_{3} / T_{2}$ :

$$
\begin{aligned}
& T_{0}=1, \\
& T_{1}=\frac{2\left(k^{2}+l^{2}\right)}{R}, \\
& T_{2} / T_{1}=\frac{2 k^{2} l^{2}}{R^{2}}, \\
& T_{3} / T_{2}=a_{3}=\frac{\left(k^{2}-l^{2}\right) A_{i}}{R\left(k^{2}+l^{2}\right)}\left(4 k^{2} A_{i}+2 m l\right) .
\end{aligned}
$$

Once we have constructed this sequence we can determine the character of the eigenvalues in this way (RouthHurwitz criterion): The number of roots with positive real parts of a real algebraic equation is equal to the number of sign changes in the sequence (11) above. [Our Eq. (8) is a real algebraic equation because all the coefficients of $\underline{\lambda}$ are real. This in turn is true because only real equilibrium points $A_{i}, B_{i}$, and $C_{i}$ are relevant.]

In (11), with real $k$ and $l$ and positive $R$, it is easy to see that the first three terms of the sequence are all positive. Therefore, the Routh-Hurwitz criterion tells us that (1) if the fourth term in the sequence is also positive, there are no changes in sign of the sequence and no positive real parts of the eigenvalues; and (2) if the fourth term in the sequence is negative, there will be one sign change in the sequence and one positive real part of the eigenvalue.

Examining $T_{3} / T_{2}$ in (11) we see that the factor outside the parentheses is always positive since $A_{i}$ is always positive when $A_{i}$ is real. The factor inside the parentheses can be positive, negative, or zero. We find that this factor is only zero when

$$
R=\left|\frac{2\left(k^{2}+l^{2}\right) k^{2}}{m\left(k^{2}-l^{2}\right)^{1 / 2}}\right|=7.59 \cdots,
$$

which is identical to $R_{c}$ in (3). Stability of the equilibria can therefore only change at $R=R_{c}$. Thus we have the following cases:

Case I: $R=R_{c}$. Then $P_{1}=P_{3}$ and $P_{2}=P_{4}$ and $a_{3}$ is zero, implying that one eigenvalue [root of (8)] is zero, implying the other two eigenvalues [roots of (8)] have negative real part. So we know the character of the eigenvalues. (By the way, since one eigenvalue is zero in this case we cannot determine the stability of the nonorigin equilibrium points.)

Case II: $R<R_{c}$. In this case the origin is the only real equilibrium point. The origin is asymptotically stable.

Case III: $R>R_{c}$. If $A_{i}=A_{1}$ or $A_{i}=A_{2}$ then $a_{3}$ is positive, so all four terms in the sequence (11) are positive, so there are no sign changes and so no positive real parts of eigenvalues. Since $a_{0}>0$, all eigenvalues have negative real parts. Therefore $A_{1}$ and $A_{2}$ are asymptotically stable. If $A_{i}=A_{3}$ or $A_{i}=A_{4}$ then $a_{3}$ is negative, so the fourth term of the sequence (11) is negative, so there is one sign change and so one positive real part of eigenvalues. Therefore $A_{3}$ and $A_{4}$ are unstable. ${ }^{5}$ Again, we have determined the character of the eigenvalues.

\section{CONCLUSIONS}

We have seen that we can easily find the character of the eigenvalues of the matrix of the linearized system associated with a system of nonlinear ordinary differential equations. There is a step-by-step procedure which uses the Routh-Hurwitz criterion in determining the character of eigenvalues. This procedure would prove useful in any study which involves examining the eigenvalues of a system, especially a nonlinear system. The procedure is simple enough that it could be adapted to computer codes, without great loss of performance.

We wish to thank Professor Gerasimos Ladas for a suggestion which led to this study.
${ }^{1}$ Martin Braun, Differential Equations and Their Applications, 3rd ed. (Springer-Verlag, New York, 1983).

${ }^{2}$ Granino A. Korn and Theresa M. Korn, Mathematical Handbook for Scientists and Engineers, 2nd ed. (McGraw-Hill, New York, 1968), p. 17.

${ }^{3}$ Edmund X. DeJesus and Charles Kaufman, Phys. Rev. A 31,
903 (1985).

${ }^{4}$ Granino A. Korn and Theresa M. Korn, Ref. 2, p. 23

${ }^{5}$ However, $A_{3}$ and $A_{4}$ are nonstable in the sense of Sparrow, due to the negative divergence of the flow for this system. C. Sparrow, The Lorenz Equations: Bifurcations, Chaos, and Strange Attractors (Springer-Verlag, New York, 1982), p. 9. 